\title{
Die Hausärzte sterben aus: Na und?
}

\section{Daniel Oertle}

Dr. med., Hausarzt in Zürich-Albisrieden, Mitglied FMH

$\mathrm{Ab}$ und zu stirbt eine Tierart aus: Der Dodo ist ausgestorben, der Säbelzahntiger und die karibische Spitzmaus. Und jetzt der Hausarzt. Das scheint traurig, ist jedoch ein natürlicher Schritt der Evolution, wie das Darwin beschreibt: Der Hausarzt stirbt aus, weil sich die Welt um ihn verändert hat und es ihn nicht mehr braucht. Seine Zeit ist um.

Ich gebe Ihnen drei Beispiele.

Herr L. ist zwischen dreissig und vierzig und also ehrgeizig, Bankangestellter, fällt vom Bike und verdreht sich bitzeli das Knie. Herr L. geht zum Hausarzt, der eine Zerrung des Innenbandes diagnostiziert. Er verschreibt Pasta boli, eine elastische Binde, ein paar Tage Schonung und kostet 56.30 Franken. Zwei Tage später erhält der Hausarzt Post von der ComprehensiveSportsClinic CSC: Im MRI wird eine Zerrung des Innenbandes mit diskretem Erguss und eine alte Läsion des inneren Meniskus beschrieben. Etwas später ruft ein erboster Herr L. an, der Hausarzt hätte die Meniskusläsion übersehen und von nun an werde er sich immer direkt in der CSC behandeln lassen, dort sei man kompetenter und hätte sofort ein MRI gemacht. Übrigens hätte man

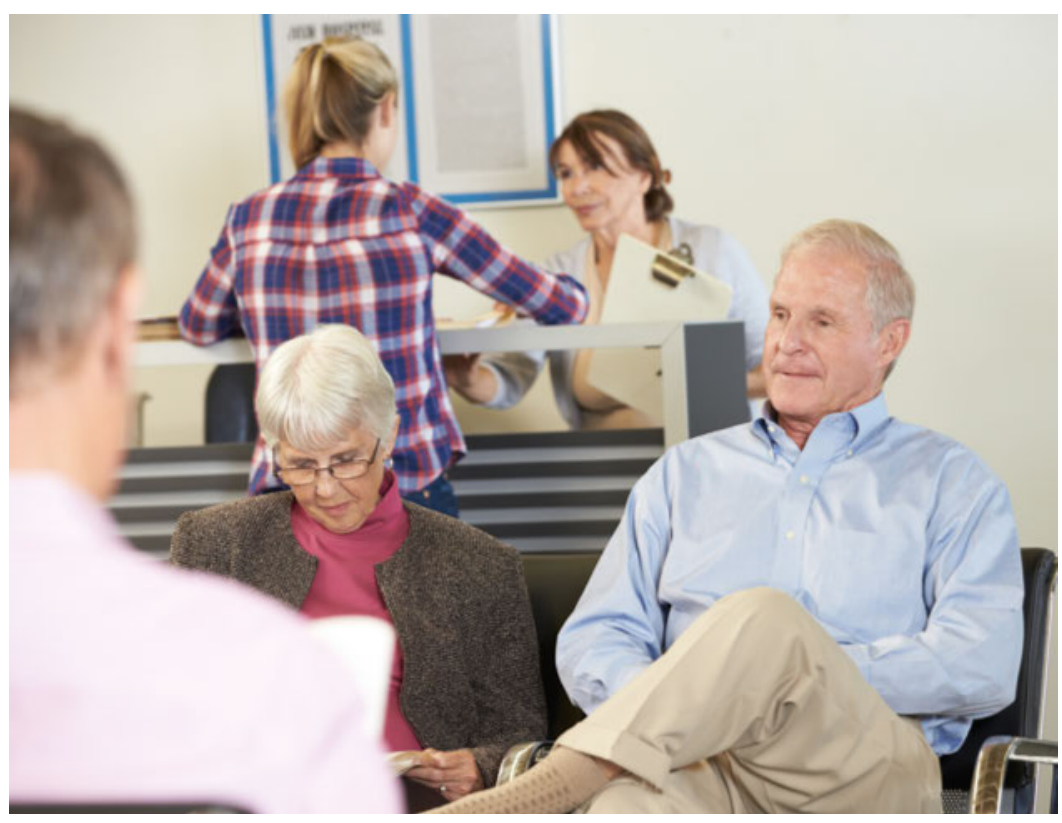

Volle Wartezimmer sind bei den Patienten von heute nicht mehr gern gesehen.

\section{Les médecins de famille sont en voie de disparition. Et alors?}

Les patients d'aujourd'hui préfèrent les cabinets ouverts $24 \mathrm{~h} / 24 \mathrm{n}$ 'exigeant pas de prise de rendez-vous ou les centres médicaux spécialisés. La médecine «instantanée» est-elle une menace pour les médecins de famille? Dans son article, Daniel Oertle décrit ce phénomène d'une plume acérée et nous fait réfléchir à l'importance du travail des médecins de famille dans la société.

ihm statt der elastischen Binde einen Aircast und vier Tage Arbeitsunfähigkeit verschrieben, dazu einen Blutverdünner. Der Hausarzt will auf den Unterschied zwischen einer alten und einer frischen Meniskusläsion hinweisen, lässt das aber bleiben und verabschiedet sich freundlich von Herrn L., den er kürzlich durch eine mühselige Scheidung begleitet hatte.

Die junge Frau, die der Hausarzt seit ihrer Kleinstkinderzeit kennt, ruft an, sie verspüre häufigen Harndrang und ein Brennen beim Wasserlassen. Die Praxisassistentin gibt ihr gleichentags einen Termin, zu dem die junge Frau nicht erscheint. Dafür bekommt der Hausarzt ein Mail der jungen Frau, sie sei bei einem InstantMedicalCheckPoint IMCP vorbeigekommen und habe dort sofort einen Termin bekommen. Man habe sie wirklich gründlich untersucht, hätte nicht nur den Urin, nein auch das Blut gecheckt, und ein junger Arzt hätte sogar einen Ultraschall gemacht - der Service im IMCP sei super und sie wolle sich jetzt immer dort behandeln lassen. Der Hausarzt klappt das Mail weg, telefoniert seinem Praxiskumpel in dessen Sprechstunde und zusammen trinken sie notfallmässig Kaffee.

Die ältere Frau ist oft beim Hausarzt, oh je, geplagt von vielen Ängsten, gebeutelt von einem strengem Leben mit eigenem Geschäft und der geistig behinderten Tochter. Diesmal ist sie überzeugt, sie hätte ein Pankreas-Karzinom, eines im Anfangsstadium. Genau so sei es bei ihrer Nachbarin gewesen, die Symptome seien am Anfang nicht wahrnehmbar gewesen - und 
als der Hausarzt endlich gehandelt habe, sei es zu spät gewesen, und jetzt sei die Nachbarin tot. Nein, so weit wolle sie es nicht kommen lassen: Sie wolle jetzt ein MRI des ganzen Körpers. Der Hausarzt gibt so etwas wie einen Seufzer von sich, setzt sich in seinem Stuhl besser hin und redet lange mit der Frau. Es seien bei ihr nun wirklich gar keine Anzeichen für ein PankreasKarzinom vorhanden und im Internet stünde halt alles Richtige, aber auch alles Falsche, und der Doktor im Fernsehen wisse auch nicht alles etc. etc. Die Frau scheint beruhigt, aber der Stundenplan ist für diesen

\section{Der Neue Patient (DNP) will subito einen Termin, beim Hausarzt oder in einem IMCP, egal: subito.}

Morgen ganz aus den Fugen. Der Hausarzt verabschiedet die Frau, tritt auf die Veranda und gibt dem blauen Hibiskus etwas Wasser.

Zwei Wochen später sitzt die Frau wieder da und bringt einen dicken Umschlag mit, den Bericht des Check-upCenters CUC. Sie habe dort zwischen drei verschiedenen Check-ups wählen können: dem Executive, dem Executive plus und dem Corporated Check-up. Sie habe den mittleren gewählt und hält dem Hausarzt einen Packen Papier hin. Er blättert diesen hastig durch und erkundigt sich höflich nach den Kosten. Nun, die Krankenkasse übernehme einen Teil, sie habe eine Zusatzversicherung - den Rest müsse sie selber bezahlen. (Der Hausarzt erinnert sich an ein Gespräch mit der Frau, dass das Haushaltsgeld manchmal kaum reiche.) Die Ärzte vom CUC hätten nichts gefunden ausser dem da: Auf Seite 3 aller Laborwerte ist hinter der Zahl für «Selen» ein Stern gesetzt, und die Frau hat «Selen» mit gelbem Leuchtstift hervorgehoben. Der Hausarzt: «Nun ja, ähm, Selen zu tief, also äh, ein Selenmangel, Selen, so so ...» Die Frau: Ob er das wirklich nicht wisse, deswegen sei sie heute da, sie habe einen Selenmangel und jetzt könne er ihr nicht einmal helfen - also diese Konsultation wolle sie dann nicht bezahlen. Der Hausarzt verschweigt, dass auch hinter dem Wert für «Molybdän» ein Stern gesetzt ist, und verabschiedet die Frau freundlich.

Lieber Leser, Sie finden die Beispiele unglaubwürdig, übertrieben? Das könnte daran liegen, dass Sie kein Hausarzt sind. Alle Hausärzte können solche Geschichten erzählen. Der Neue Patient (DNP) will subito einen Termin, beim Hausarzt oder in einem IMCP, egal: subito. Am besten lässt sich der Arzttermin mit dem übrigen Shopping verbinden - und seit die Lebensmittel-Grossverteiler im Gesundheitsmarkt mitmischen, lässt sich der Arztbesuch bequem über die Cumulus-Karte $a b$ buchen. DNP will subito eine genaue Diagnose, am bes- ten mit Bildgebung. Eine umschreibende Beurteilung genügt nicht - dabei läge gerade darin die Stärke der Hausarztmedizin: Es gilt, eine essentielle Krankheit oder eine schwere Verletzung auszuschliessen, die eine sofortige Therapie nötig machten - um sich dann in folgenden Konsultationen der Diagnose anzunähern, während dazwischen die Zeit heilt. DNP hat diese Zeit nicht, sein InstantMedicalCheckPoint-Adviser IMCPA ist über Facebook, Twitter und Whatsapp 24/7 erreichbar. DNP ist unbekümmert über die Kosten, die er verursacht - schliesslich bezahle er Krankenkassenprämien.

Noch vor 20 Jahren gab es keine InstantMedicalCheckPoints IMCP, und niemand vermisste sie. Das Bedürfnis nach Instant-Medizin musste zuerst geschaffen und will jetzt befriedigt werden. IMCP waren für Notfälle da - und jetzt wird der Begriff "Notfall» nach Belieben gedehnt: Ein verdrehtes Knie ist ein Notfall, eine Blasenentzündung auch - auch ein Check-up kann notfallmässig durchgeführt werden. Nachdem alle Bahnhöfe mit IMCPs bestückt sind, werden solche auch an grösseren Tramhaltestellen eingerichtet, und die Spitäler blähen ihre Notfallstationen auf. Krankenkassen, Lebensmittel-Grossverteiler und internationale Health-Management-Konzerne mischen mit, das Geschäft läuft. Hausärzte sind in diesem Business überflüssig, ihre bedächtige, sorgfältige Medizin behindert das flotte Wachstum des medizinisch-industriellen Komplexes, der zu den bestprosperierenden Sparten unserer Volkswirtschaft gehört.

\section{Die allerletzten Hausärzte werden in} geographischen Randgebieten in freier Wildbahn zu beobachten sein.

Den verbliebenen Hausärzten wird die Arbeit noch nicht ausgehen. Es gibt noch Die Alten Patienten (DAP), die Geduld haben, auf einen Termin zu warten, und akzeptieren, dass eine Diagnose manchmal erst im Verlauf einer Krankheit gestellt werden kann. DAP haben erfahren, dass viele Befindlichkeitsstörungen keinen lateinischen Namen haben und nach ein paar Tagen aggressiven Abwartens spurlos verschwinden. Die allerletzten Hausärzte werden in geographischen Randgebieten in freier Wildbahn zu beobachten sein, bevor dann diese Randgebiete geschlossen werden, wie Politiker dies fordern. Dann wird der Hausarzt aussterben wie vor ihm der Dodo, der Säbelzahntiger und die karibische Spitzmaus.

\section{Bildnachweis}

(C) Monkeybusinessimages | Dreamstime.com
Dennlerstrasse 25

daniel.oertle[at]hin.ch 